\title{
Criminologie
}

\section{La Société de criminologie du Québec : historique, activités et perspectives d'avenir}

\section{José Rico}

Volume 10, numéro 2, 1977

La criminologie au Québec

URI : https://id.erudit.org/iderudit/017073ar

DOI : https://doi.org/10.7202/017073ar

Aller au sommaire du numéro

Éditeur(s)

Les Presses de l'Université de Montréal

ISSN

0316-0041 (imprimé)

1492-1367 (numérique)

Découvrir la revue

Citer cet article

Rico, J. (1977). La Société de criminologie du Québec : historique, activités et perspectives d'avenir. Criminologie, 10(2), 55-61.

https://doi.org/10.7202/017073ar d'utilisation que vous pouvez consulter en ligne.

https://apropos.erudit.org/fr/usagers/politique-dutilisation/ 
LA SOCIETE DE CRIMINOLOGIE DU QUEBEC : HISTORIQUE, ACTIVITÉS

PERSPECTIVES D'AVENIR

José Rico

\section{HISTORIQUE}

La Société de criminologie du Québec fut fondée, en juin 1960, par un groupe de personnes préoccupées par laugmentation constante de la criminalité - adulte et juvénile - et par les problèmes que les formes anciennes et nouvelles posaient à ceux qui ont la responsabilité de la protection de la société. Ces personnes venaient de tous les horizons policiers et psychiatres, administrateurs de pénitenciers et éducateurs, universitaires et magistrats, membres du barreau et du clerge, etc. - et avaient un souci commun : celui de dépasser, dans la lutte contre la criminalité, les cloisons étanches entre professions et services, regroupant tous ceux qui, à quelque titre que ce soit, s'intéressaient au phénomène de la criminalité et de la déviance. Tous avaient réalisé que, seuls, ils étaient impuissants à résoudre les problèmes qui se posaient à eux; ils sentaient également que sans études approfondies et sans une information adéquate de l'opinion publique sur ces questions de grande complexité, aucun progrès véritable ne pouvait être envisage dans leurs méthodes d'administration de la justice.

Un cadre favorable au dialogue et aux échanges, dans une perspective d'étude scientifique et de confrontation loyale d'opinions sur des sujets prêtant à controverse, fut ajnsi cré et instauré.

\section{Organisation}

Depuis sa création, la Sociêté de criminologie du Québec se fixa les objectifs suivants :

1) grouper, dans la province de Québec, les personnes qui s'intéressent à l'étude et à la solution des problèmes que posent la criminalité et la délinquance ;

2) creer dans cette province, un organisme permanent capable d'assurer les contacts et d'harmoniser la collaboration entre les personnes et les groupes qui se vouent, dans les divers secteurs, à cette étude et à ces solutions ;

3) Étudier les problèmes posés par l'administration de la justice, des Etablissements pénitentiaires, des ceuvres de rébabilitation, des services de probation et de protection et de tout autre groupe ou service et promouvoir, par des suggestions appropriées, les réformes pénales nécessaires ainsi qu'une orientation toujours plus scientifique et plus humaine de la répression de la criminalité et des mesures de réhabilitation; appuyer les organismes qui, suivant leur profession ou autrement, s'intéressent à ces mêmes questions ;

4) permettre, par des colloques et des conférences, à des spécialistes de ces questions, de présenter et de faire valoir leurs observations au sujet de chacun des aspects de la criminalité qui préoccupent les membres de la Société ;

5) developper, dans tous les milieux de la province de Québec, une meilleure connaissance des problèmes de la criminalité et par là obtenir 
d'eux la collaboration active qu'ils peuvent apporter à la société et déterminer la forme que doit prendre cette collaboration ;

6) s'occuper de la prophylaxie ou des mesures préventives, en suggérant ou appuyant les initiatives qui, à tous les paliers de la vie sociale, tendent à prevenir le crime ;

7) encourager la recherche criminologique ;

8) promouvoir la formation d'un personnel qualifie dans l'ensemble des secteurs où l'on s'occupe de la délinquance juvénile ou adulte.

Créée au profit de la collectivité québécoise et appelée à exercer ses activités au sein de celle-ci, notre Société s'inspire, dans tout ce qu'elle entreprend ou soutient, du principe suivant : en aucun cas la défense sociale ne pourra être élaborée et développée au détriment des droits fondamentaux de la personne humaine, formulés dans la Déclaration universelle des droits de l'homme (Paris, 1948).

Les règlements de la Société furent adoptés en 1960 et amendés en 1967. Le premier président fut le R.P. Noël Mailloux, professeur de psychologie à l'Université de Montréal, personnalité de premier plan aussi bien au Canada que sur la scène internationale dans le champ de la réducation et de la délinquance juvénile. Le premier secrétaire général de la Société fut le professeur Denis Szabo, directeur à l'époque, du département de criminologie à l'Université de Montréal. Les autres présidents de la Société ont été : le docteur Bruno Cormier, professeur de psychiatrie médico-légale à l'Université McGill, bien connu par ses travaux sur les populations penitentiaires (1962-1964); l'honorable Roger Ouimet, juge à la Cour supérieure de Montréal, qui, en 1965, quitta la présidence pour assumer celle du Comité canadien de la réforme pénale et correctionnelle; Me Joseph Cohen, c.r., doyen des avocats criminalistes du Québec (19651969) ; M. Guy Guérin, juge à la Cour des Sessions de la paix de Montréal, professeur à la faculté de đroit de l'Université de Montréal et président de la Conférence des juges du Québec (1969-1974); l'honorable Antonio Lamer, juge à la Cour supérieure de Montréal et président de la Com. mission de reforme du droit du Canada (1974-1976); et $M$. Marcel Trahan, juge à la Cour de Bien-Être social de Montréal et pionnier de la rfforme de la justice pour mineurs dans notre province.

La Societé compte actuellement environ 500 membres, qui proviennent de milieux fort divers : barreau, magistrature, universite, prisons et pénitenciers, police, services de probation et de libération conditionnelle, centres de traitement psychiatrique, services de réhabilitation, etc.

La Société de criminologie du Québec a toujours favorisé la création et la formation de sections régionales, afin que ses membres puissent rendre des services plus adéquats dans leurs régions respectives. A l'heure actuelle, il existe des sections régionales dans neuf des dix régions administratives du Québec (le Nouveau Québec n'a pas encore de service régional). Chaque section régionale jouit d'une autonomie presque complète, qui lui permet de prendre ses propres initiatives. Le Conseil provincial assume les fonctions de coordination et de planification des sections régionales, organise les activités sur le plan provincial et sert de liaison avec les autres associations ainsi qu'avec le gouvernement.

\section{ACTIVITÉS}

Les activités de la Société peuvent se regrouper sous les rubriques suivantes : 1) conférences publiques et tables rondes; 2) colloques de 
recherche; 3) congrès; 4) publications; 5) action sur la communaute; et 6) attribution de prix.

1) Conférences publiques et tables rondes :

De nombreuses conférences, panels et tables rondes ont eu lieu dans les principales villes et régions du Québec. Voici, à titre indicatif, quelquesuns des sujets abordés : * l'égalité des citoyens devant la loi : faut-il réformer l'assistance judiciaire? „, \& la Commission de police de l'Ontario ", l'institution pénale dans un monde en évolution », clibertés individuelles et justice répressive : examen du rôle de la police, de la poursuite et des tribunaux en France et au Canada ,, e la responsabilité diminuée et la politique criminelle , " loi-modèle sur la sentence *, la prostitution et sa prévention sur le plan policier ", a le ministère public s, crime organisé et pouvoirs de police : un défi pour la protection sociale dans une société libre ", "les pratiques policières au Canada et aux Etats-Unis,, la participation du public pour permettre au détenu une activité utile dans le milieu carcéral et sa réintégration sur le marché du travail à sa libération *, * la justice est-elle la même pour les riches et pour les pauvres ? ", I'usage des hallucinogènes et ses implications criminologiques", "l'école et le policier ", e les vols à main armée dans les banques ", "les facteurs de détérioration de la famille et la délinquance juvénile s, a la publicité dans les affaires criminelles ", eles droits de l'homme devant la justice pénale ,, - les mauvais traitements des enfants ", " le vol à l'étalage ", etc.

En moyenne, une centaine de personnes assistent à chacune de ces séances et la presse en assure une diffusion assez large. Bien des idées ont commencé à pénétrer dans notre milieu à partir des tables rondes de la Société. Citons, entre autres, les problèmes du juge d'instruction, de la compensation des victimes d'actes de violence, de l'élargissement des cadres de l'assistance judiciaire, de la création d'un conseil consultatif en matière de justice criminelle, etc.

\section{2) Colloques de recherche :}

Sept colloques de recherche ont eu lieu entre 1959 et 1971. En général, ces colloques ont réuni pendant quelques jours de deux à trois cents participants du Québec, de l'Ontario, des autres provinces canadiennes et, parfois, des Etats-Unis. Les sujets abordés couvrent la plupart des champs de l'étiologie de la conduite criminelle ainsi que l'étude scientifique des problèmes de ladministration de la justice pénale.

Voici, à titre d'exemple, les problèmes traités durant les colloques de 1967 et 1969 : le criminel d'habitude, l'évaluation de l'efficacité du traitement dans une École de protection, les délits de violence à Montréal, moralité adolescente et structure sociale, les problèmes suscités par la recherche dans le traitement institutionnel, l'homosexualité en milieu carcéral, les délits politiques et idéologiques, tendances et évolution de la délinquance juvénile à Montréal, modèles de consommation d'alcool chez les délinquants et les non-délinquants, enquête d'opinion publique sur la police au Québec, étude transculturelle de la criminalité féminine, éducation sexuelle et délinquance juvénile, recherche à long terme sur la criminalité se développant à un âge avancé, la défense sociale et les droits de l'homme, le traitement des criminels malades mentaux. Le dernier colloque, en 1971, a été consacré à la recherche appliquée en criminologie.

Ces manifestations sont bilingues et la traduction simultanée est assurée; la criminologie s'est ainsi taillé une place de choix dans le 
concert des disciplines scientifiques au Canada et son rayonnement dépasse même nos frontières.

\section{3) Congrès :}

Des congrès provinciaux sont organisés depuis 1965 , alternant ainsi avec les colloques de recherche. Lors de ces congrès, les principaux problèmes de politique criminelle sont abordés; l'on procède de la sorte à une confrontation des chercheurs et des praticiens au sujet des diverses manifestations de la conduite criminelle et du fonctionnement des différentes branches de l'administration de la justice.

C'est ainsi que, lors du premier congrès, qui groupa plus de quatre cents personnes et qui eut lieu à l'Université de Montréal en avril 1966, les problemes des prisons et des centres de réeducation pour mineurs furent examinés. Le deuxième congrès se réunit à Sherbrooke, en mars 1968 , et est consacré à la probation. Le troisième congrès eut lieu à Québec, en 1972 ; il avait comme thème la police. Le $14^{\mathrm{e}}$ congrès ${ }^{1}$ organisé à Montréal en mars 1973, comprenait quatre sessions, qui traitèrent respectivement des droits de l'homme et des droits des détenus, du rôle du criminologue dans la société, de la police et du pouvoir politique municipal et de la prévention de la délinquance à l'école et dans la communauté. Le $15^{\mathrm{e}}$ congrès (Montréal, mars 1974) adopta lui aussi la formule de quatre sessions, présentées de la façon suivante : les établissements de détention (provinciaux) et l'administration de la justice au Québec, les institutions pour mineurs délinquants doivent-elle disparaître?, où va la libération conditionnelle ? et la justice criminelle est-elle accessible à tous? Finalement, le $17^{\mathrm{e}}$ congrès, intitulé * La justice et l'injustice au Québec *, eut lieu à Montréal, en mars 1976. Deux tables rondes établirent le bilan des derniers rapports publiés par les différents ministères, commissions d'enquête et de réforme qui se sont penchés, au cours des dernières années, sur plusieurs aspects de la criminalité et de l'administration de la justice aussi bien au Québec qu'au Canada et analysèrent l'opinion publique vis-à-vis des problèmes de la criminalité et de la justice ainsi que le rôle joué par les masses média dans l'éducation du public. Des thèmes plus précis fla déjudiciarisation, la délinquance des mineurs, la police, la détermination de la sentence, les peines privatives de liberté, les alternatives à la prison, les projets novateurs et communautaires) firent l'objet de débats dans sept ateliers de travail.

Une véritable prise de conscience des problèmes criminologiques naît lors de pareilles manifestations et l'opinion publique commence à mesurer les dimensions du phénomène criminel et réalise la nécessité de rechercher de meilleurs moyens de protection.

4) Publications :

De juin 1961 à septembre 1966 fut publié un Bulletin, qui paraissait trois fois l'an et contenait aussi bien des articles de fond que des nouvelles concernant la vie de la Société et les principaux événements de caractère criminologique. En 1967, cette publication devint un Bulletin de nouvelles, dont le but était de servir d'agent d'information et de liaison pour tous les membres de la Société. Quant aux articles scientifiques portant sur des travaux de recherche, ils devaient paraître dans la revue du département

1. A partir de 1973 , le numero d'ordro des congrès a ete change, afin de les faire coüncider avec les années d'existence de la Société. 
de criminologie de l'Université de Montréal, Acta Criminologica, dont le premier volume fut publie en janvier 1968 par les Presses de l’Université de Montréal.

Cependant, la publication du Bulletin de nouvelles fut interrompue vers la fin de l'année 1969. En ce qui concerne Acta Criminologica, cette revue continue à paraître régulièrement. En 1975, son nom et son contenu ont été changés ; désormais, son titre est Criminologie et son contenu essaie de refléter prioritairement les travaux et les préoccupations propres à la communauté intellectuelle québécoise et de présenter aux professionnels de la justice les résultats des recherches criminologiques effectuées au Québec ainsi que les réflexions des théoriciens et des praticiens de la justice pénale, le tout sous une forme plus accessible que par le passé. En principe, chaque volume porte sur un thème spécifique. Ont été traités jusqu'à présent les sujets suivants: la délinquance juvénile (1975), l'emprisonnement (1976) et la criminalité des affaires (1977).

\section{5) Action sur la communauté :}

La diffusion faite par la presse écrite, parlée et télévisée des congrès, conférences et tables rondes organisés par la Société représente une première action d'ordre général sur la collectivité québécoise, qui est déjà bien familiarisée avec nos activités. À cela il faut ajouter le fait qu'un bon nombre de nos membres ont été et sont appelés à siéger sur différents comités gouvernementaux ou autres.

De façon spécifique, la section de Québec s'est servie de la télévision pour exposer les difficultés que rencontrent les ex-détenus en se cherchant un emploi et dans les Cantons de l'Est, on a également utilisé la télévision pour sensibiliser la population à des sujets d'actualité en matière de criminologie. Par ailleurs, des initiatives sont en marche, dans les régions de Montréal et de l'Outaouais, pour créer des comités de citoyens ; ceux-ci auraient pour but d'évaluer l'efficacité des méthodes employées par l'administration de la justice et de suggérer les réformes adéquates.

Finalement, dans certains C.E.G.E.P. de la province sont donnés des cours en criminologie à l'intérieur de plusieurs concentrations : "sciences policières *, - techniques correctionnelles *, * assistance sociale *, etc. A l'Université de Montréal, des cours de criminologie sont également offerts dans le cadre de l'éducation permanente.

6) Attribution de prix :

En 1964, deux prix ont été crés par le Conseil d'administration de la Société afin de contribuer à développer une tradition d'excellence dans notre milieu et également pour permettre d'honorer ceux qui ont fait une contribution particulière au progrès de la discipline criminologique.

Le prix Archambault-Fauteux, ainsi appelé en mémoire de deux éminents magistrats qui ont marqué l'histoire de l'administration de la justice dans notre pays, est destiné à honorer une personnalité dont l'œuvre constitue une contribution importante à l'administration de la justice (cours, barreau, police, etc.). Le palmarès des récipiendaires est, en quelque sorte, une récapitulation des événements qui ont influencé le progrès des idées criminologiques depuis le début des années 60. Le cardinal PaulÉmile Léger fut parmi les premiers à encourager des réformes dans le domaine de la délinquance juvénile; le sénateur Thérèse Casgrain, dont le nom est à jamais associé à la lutte contre les injustices sociales, représente, avec le professeur Maxwell Cohen, responsable d'une commission 
d'enquête sur la littérature propageant la haine raciale, la tradition du respect des droits de I'homme, y compris de ceux qui sont les plus rejetes par la majorité de la société. Le bâtonnier Jean Martineau, ainsi que les juges Roger Ouimet et Yves Prévost, ont été des membres du barreau et de la magistrature qui n'ont pas hésité à se consacrer à la réforme de la justice et du système correctionnel du Canada et du Québec. M. Paul-André Rivard a grandement contribué à l'instauration et au développement des services de probation dans notre province. Le dernier prix a été décerné à M. Jean-Pierre Charbonneau, pour ses travaux de recherche sur le crime organisé.

Etabli pour distinguer des jeunes chercheurs dont la contribution a la science criminologique a été remarquée, le prix Beccaria a déjà été accordé à Mlle Thérèse Limoges, pour son livre sur la prostitution à Montréal; au docteur Richard Michaud, pour ses travaux en criminologie clinique; aux professeurs Ezzat A. Fattah pour ses études sur la victimologie, Brian Grossman pour son livre sur le procureur de la Couronne, Denis Gagné, coauteur du livre l'Adolescent et la société et Marc Le Blanc, auteur de la monographie Une analyse stigmatique de la réaction sociale da la délinquance juvénile, ainsi qu’à M. Raymond Boyer, pour son livre sur le crime et le châtiment au Canada français ainsi que pour son activité au sein du Comité de défense des droits des détenus.

A ces prix, on a ajouté récemment le prix Denis Gagné, décerné aux personnes ayant ceuvré dans le secteur de la prévention de la délinquance. Les premiers récipiendaires ont été conjointement MM. Claude Bussières et Denis Boivin.

\section{PERSPECTIVES D'AVENIR}

L'examen des activités de notre Société depuis 1960 fait surgir le problème de l'avenir. Après une période de mise en marche, de déchiffrage et d'organisation, nous somnes actuellement à l'étape de la consolidation. Celle-ci se présente sur deux plans : celui des activités et celui de l'organisation des services.

\section{1) Activités :}

Pour maintenir les activités actuelles et pour en augmenter la qualité et le rayonnement, une évaluation approfondie doit être effectuée. Un effort particulier a déjà été fait pour accroître le rayonnement de la Société dans les milieux anglophones du Québec. Les sections régionales déploient depuis quelques années une activité considérable; en effet, des conférences publiques suivies de débats, des tables rondes, des colloques, etc., ont lieu chaque année dans les diverses contrées de la province. Il est cependant indispensable que la Société, dont la majorité des membres sont montréalais, puisse aider ses sections à s'organiser et à développer une activitó régionale de qualité, en mettant en branle le même mouvement d'information, d'éducation et d'étude qui a déjà porté fruit à Montréal. Nos congrès commencent par ailleurs à avoir lieu dans les diverses parties de la province, permettant ainsi d'envisager tous les problèmes criminologiques dans leur ampleur régionale.

Le Bulletin de nouvelles devrait paraître de nouveau plusieurs fois par anné, donnant des informations régulières sur la vie de la Société et de ses sections régionales, ainsi que sur les événements d'ordre criminologique survenus au Québec, au Canada et dans le monde. 
Les actes de nos congrès et de nos colloques de recherche devraient être régulièrement publiés. Grâce aux subventions occasionnelles, nous avons pu assurer la parution des actes de plusieurs de ces congrès et colloques. Les articles publiés dans les revues criminologiques du monde entier ont contribué au rayonnement culturel de notre province.

Le programme des activités d'une année devrait être déterminé à l'avance et porté à la connaissance des membres de la Société et du public; il en résultera une assistance plus suivie et une meilleure planification des sujets à aborder.

Des mémoires sur les questions d'actualité scientifique et de politique criminelle devraient être préparés par le Conseil d'administration; la Société pourrait ainsi mieux accomplir son rôle d'informateur des pouvoirs et de l'opinion publics.

\section{2) Organisation des services :}

Depuis sa fonđation, la Société n'a pu compter que sur les revenus provenant de la cotisation de ses membres, ainsi que du bénévolat de ceux-ci. Ce dernier a été considérable; sans ce dévouement exemplaire de beaucoup de personnes, la Société n'aurait pas pu exister. Mais, étant donné sa croissance, l'extension de ses activités et l'importance des services que la communauté attend d'elle, la Société ne peut plus faire face à ses responsabilités avec les mêmes moyens qui furent les siens depuis sa fondation. Il semble indispensable qu'un secrétariat permanent continue à assurer l'infrastructure technique nécessaire pour mener à bien les activités ci-haut énumérées et que des fonds soient disponibles pour assurer l'impression régulière du Bulletin et des actes des congrès et des colloques de recherche. 